\title{
Amélioration du transfert convectif par l'ajout d'un obstacle poreux dans une confuguration annulaire
}

\author{
Khadija Choukairy a et Rachid Bennacer \\ LEEVAM-LEEE, 5 mail Gay Lussac, Université de Cergy, 95031 Cergy-Pontoise Cedex, France
}

Reçu le 7 juin 2005, accepté le 25 avril 2006

\begin{abstract}
Résumé - Les transferts par convection naturelle sont fréquemment utilisés dans les différents procédés et sont également rencontrés dans diverses situations dans la nature. Afin d'améliorer ces transferts sans modifier la géométrie, il est possible de perturber l'écoulement d'origine par un obstacle poreux le long du trajet de l'écoulement principal. Cet obstacle modifie la structure de l'écoulement et affecte les transferts locaux. Cette modification est plus prononcée dans une configuration cylindrique au vu de la dissymétrie des écoulements résultants. La variation temporelle du transfert de chaleur est analysée pour différentes courbures et différentes valeurs du nombre de Darcy dans le milieu poreux. Cela nous permettra d'étudier les trois configurations : l'absence de l'obstacle (cas fluide), sa présence (bloc solide) et le cas intermédiaire du bloc poreux. Les conditions nécessaires pour améliorer le transfert dans chaque configuration sont illustrées.
\end{abstract}

Mots clés : Convection naturelle / obstacle poreux / cavité annulaire / transfert de chaleur / courbure

\begin{abstract}
Improvement of the convective transfer by the intrusion of an annular configuration. The heat transfer by natural convection are frequently used in the various processes and is also met in various situations in nature. In order to improve these transfers without modifying the geometry, it is possible to disturb the flow of origin by a porous obstacle along the way of the principal flow. This obstacle modifies the structure of the flow and affects the local transfers. This modification is more marked in a cylindrical configuration within sight of the dissymmetry of the resulting flows. The temporal variation of the heat transfer is analyzed for different curvatures and different values of Darcy number in the porous environment. That will enable us to study the three configurations: the absence of the obstacle (fluid case), its presence (solid block) and the intermediate case of the porous block. We illustrate the conditions necessary in order to improve the transfer in such a configuration.
\end{abstract}

Key words: Natural convection / porous obstacle / annular cavity / heat transfer / curvature

\section{Introduction}

Les écoulements de convection naturelle dans les espaces confinés et en particulier dans des cavités cylindriques, constituent depuis plusieurs décennies l'objet de nombreuses études dues au rôle important qu'ils jouent dans beaucoup d'applications techniques ou technologiques (comme le confort de bâtiment, le refroidissement des composants électroniques, les échangeurs de chaleur et dans bien d'autres domaines...).

De Vahl Davis et Thomas [1] étaient parmi les premiers à rapporter une étude numérique de la convection naturelle dans des cavités cylindriques et leurs résultats ont été prolongés par d'autres auteurs Prasad et Kulacki [2], Kumar et Kalam [3], Prasad [4].

a Auteur correspondant :

Khadija.Choukairy@iutc.u-cergy .fr
Ils ont constaté que la courbure du cylindre peut fortement affecter la structure d'écoulement et le transfert thermique dans le cylindre. Ils ont également noté la réduction de la température au centre avec l'augmentation de la courbure. Ceci est dû à la non-symétrie du transfert thermique entre les cylindres intérieur et extérieur. Les travaux mentionnés ont été présentés en régime permanent. Contrairement au régime permanent, l'étude transitoire de la convection naturelle dans une cavité a suscité beaucoup moins d'attention dans la littérature en dépit de son intérêt scientifique et technologique (collecteurs d'énergie solaire, alimentation électrique des circuits par un courant alternatif...).

Parmi les contributions effectuées dans ce cadre nous pouvons citer celles de Paterson et Imberger [5], Hyun et Lee [6] dans le cas de la cavité cartésienne. L'objectif de notre étude est de comprendre la façon dont le transfert thermique est modifié pendant la phase transitoire 


\section{Nomenclature}

\begin{tabular}{|c|c|}
\hline$A$ & Rapport de forme, $=\left(r_{0}^{\prime}-r_{\mathrm{i}}^{\prime}\right) / H$ \\
\hline$g$ & Accélération de la gravité, m.s ${ }^{-2}$ \\
\hline Gr & Nombre de Grashof, $=g \beta \Delta T H^{3} / v^{2}$ \\
\hline$H$ & Hauteur de la cavité, m \\
\hline$K$ & Perméabilité, $\mathrm{m}^{2}$ \\
\hline$L$ & Distance radiale entre les deux cylindres verticaux, $=\left(r_{0}^{\prime}-r_{\mathrm{i}}^{\prime}\right)$ \\
\hline $\mathrm{Nu}$ & Nombre de Nusselt \\
\hline$P$ & Pression adimensionnelle, $=p^{\prime} H^{2} / \rho v^{2}$ \\
\hline $\operatorname{Pr}$ & Nombre de Prandtl, $=v / \alpha$ \\
\hline$R$ & Courbure, $=L / r_{\mathrm{i}}^{\prime}$ \\
\hline$r$ & Coordonnée radiale sans dimension, $=r^{\prime} / H$ \\
\hline$r^{\prime}$ & Coordonnée radiale avec dimension, m \\
\hline$S$ & Surface du cylindre \\
\hline$\tau$ & Temps \\
\hline$u$ & Vitesse adimensionnelle dans la direction radiale, $=u^{\prime} H / v$ \\
\hline vol & Volume du cylindre \\
\hline$w$ & Vitesse adimensionnelle dans la direction axiale, $=w^{\prime} H / v$ \\
\hline$z$ & Coordonnée axiale sans dimension, $=z^{\prime} / H$ \\
\hline \multicolumn{2}{|c|}{ Symboles grecs } \\
\hline$\alpha$ & Diffusivité thermique, $\mathrm{m}^{2} \cdot \mathrm{s}^{-1}$ \\
\hline$\beta_{T}$ & Coefficient d'expansion volumétrique, $K^{-1}$ \\
\hline$\Delta T$ & Différence de température, $=\left(T_{\mathrm{c}}-T_{\mathrm{f}}\right), \mathrm{K}$ \\
\hline$\theta$ & Température adimensionnelle, $=\left(T-T_{\mathrm{f}}\right) /\left(T_{\mathrm{c}}-T_{\mathrm{f}}\right)$ \\
\hline$v$ & Viscosité cinématique, $\mathrm{m}^{2} \cdot \mathrm{s}^{-1}$ \\
\hline$\rho$ & Densité du fluide, $\mathrm{kg} \cdot \mathrm{m}^{-3}$ \\
\hline \multicolumn{2}{|c|}{ Indices } \\
\hline $\mathrm{c}$ & Chaud \\
\hline $\mathrm{f}$ & Froid \\
\hline $\mathrm{i}$ & Cylindre intérieur \\
\hline $\mathrm{o}$ & Cylindre extérieur \\
\hline
\end{tabular}

dans différentes configurations : est-il possible d'augmenter le transfert thermique en utilisant des régimes oscillatoires plutôt que l'état d'équilibre? Puisque l'augmentation de la surface d'échange induit un accroissement des transferts, on utilisera un bloc poreux. L'effet de la perméabilité du bloc poreux permettra de caractériser l'évolution temporelle des cas intermédiaires entre les cas extrêmes représentés par le cas d'un bloc solide et le cas du fluide.

\section{Formulation mathématique}

Le domaine étudié consiste en l'espace entre deux cylindres coaxiaux verticaux rempli d'air $(\operatorname{Pr}=0,7)$ dont les parois verticales sont maintenues à des températures différentes alors que les parois horizontales sont supposées adiabatiques (Fig. 1).

Les transferts radiatifs ainsi que les dissipations visqueuses sont supposés négligeables. La masse volumique dans le terme de force de volume est supposée dépendre linéairement de la température :

$$
\left(\rho-\rho_{\mathrm{o}}\right) / \rho_{\mathrm{o}}=\beta_{\mathrm{T}}\left(T-T_{\mathrm{o}}\right) \quad \text { ou } \quad \beta_{\mathrm{T}}=-1 / \rho\left(\frac{\partial \rho}{\partial T}\right)_{P}
$$

Le modèle mathématique repose sur l'hypothèse d'un fluide newtonien en écoulement laminaire, incompressible.

L'obstacle (forme annulaire) est placé à l'intérieur du domaine. Ce dernier modifie à la fois l'écoulement (obligé de le contourner) et le champ de température par la conduction supplémentaire au travers du bloc.

Pour écrire le système d'équation sous forme adimensionnelle, nous choisissons la hauteur de la cavité comme grandeur de référence $(H)$, et pour le temps et la vitesse de référence nous utilisons des grandeurs de référence basées sur la viscosité cinématique

$$
\begin{gathered}
r=r^{*} / H, \quad z=z^{*} / H, \quad \tau=\tau^{*} \nu / H^{2}, \quad u=u^{*} H / \nu, \\
w=w^{*} H / \nu, \quad \theta=\frac{T-T_{\mathrm{f}}}{T_{\mathrm{c}}-T_{\mathrm{f}}}
\end{gathered}
$$




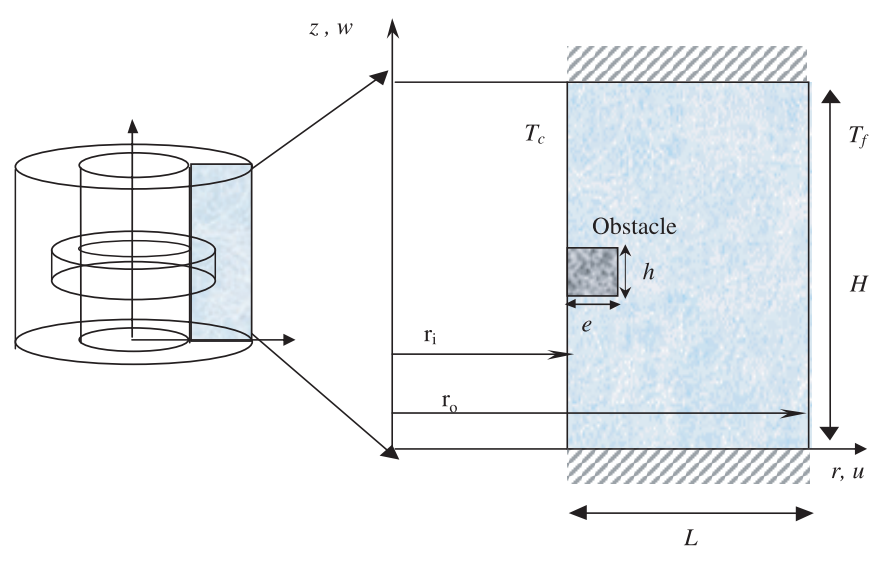

Fig. 1. Configuration étudiée : un obstacle rectangulaire est placé dans l'espace annulaire entre deux cylindres.

En termes adimensionnés, les équations de conservation s'écrivent sous la forme suivante :

- Équation de conservation de la masse

$$
\frac{1}{r} \frac{\partial(r u)}{\partial r}+\frac{\partial w}{\partial z}=0
$$

- Équations de conservation de la quantité de mouvement

$$
\begin{aligned}
& \frac{\partial w}{\partial \tau}+u \frac{\partial w}{\partial r}+w \frac{\partial w}{\partial z}=-\frac{\partial p}{\partial z}+\nabla^{2} w-\frac{w}{\mathrm{Da}}+\operatorname{Gr} \theta \\
& \frac{\partial u}{\partial \tau}+u \frac{\partial u}{\partial r}+w \frac{\partial u}{\partial z}=-\frac{\partial p}{\partial r}+\left(\nabla^{2} u-\frac{u}{r^{2}}\right)-\frac{u}{\mathrm{Da}}
\end{aligned}
$$

- Équation de conservation de l'énergie

$$
\frac{\partial \theta}{\partial \tau}+u \frac{\partial \theta}{\partial r}+w \frac{\partial \theta}{\partial z}=\frac{1}{\operatorname{Pr}} \nabla^{2} \theta
$$

Les paramètres adimensionnels qui caractérisent le problème sont les nombres classiques : le nombre de Grashof : $\mathrm{Gr}=g \beta \Delta T H^{3} / \nu^{2}$, le nombre de Prandtl $\operatorname{Pr}=\nu / \alpha$ et le nombre de Darcy Da $=K / H^{2}$.

Les conditions aux limites aux parois sont le nonglissement $(u=w=0)$ pour la dynamique et pour la thermique on impose :

$$
r=r_{\mathrm{i}} \quad \theta=1 \quad r=r_{\mathrm{o}} \quad \theta=0 \quad Z=0,1 \frac{\partial \theta}{\partial z}=0
$$

Le transfert de chaleur à travers le cylindre intérieur $(r=$ $\left.r_{\mathrm{i}}\right)$ et extérieur $\left(r=r_{\mathrm{o}}\right)$ sont exprimés en forme sans dimensions par le nombre de Nusselt :

$$
\begin{gathered}
\mathrm{Nu}_{\mathrm{i}}=\left.\int_{0}^{1} \frac{\partial \theta}{\partial r}\right|_{r=\frac{1}{R}} \mathrm{~d} z \\
\mathrm{Nu}_{\mathrm{o}}=\left.\int_{0}^{1} \frac{\partial \theta}{\partial r}\right|_{r=\frac{R+1}{R}} \mathrm{~d} z
\end{gathered}
$$

Signalons que :

$$
\frac{\mathrm{Nu}_{\mathrm{i}}}{\mathrm{Nu}_{\mathrm{o}}}=\frac{r_{\mathrm{o}}}{r_{\mathrm{i}}}=1+R
$$

\section{Procédure numérique}

Les équations couplées (1) à (4) avec les conditions aux limites sont résolues en utilisant la méthode de volumes finis. Cette méthode classique est décrite par Patankar [7]. La résolution est faite en variables primitives et le couplage vitesse-pression est résolu en utilisant l'algorithme SIMPLER. La solution est obtenue quand le maximum d'erreur pour chacune des équations est inférieur à $10^{-9}$ pour chaque pas de temps.

Afin d'assurer l'indépendance des résultats de simulations vis-à-vis des paramètres de discrétisation, une analyse de l'effet du maillage et du pas de temps a été entreprise.

Il a été montré qu'un maillage irrégulier (sinusoïdal) est nécessaire. Ce type de maillage permet de capturer les forts gradients et les couches limites près des parois des cylindres et autour de l'obstacle. Les résultats qui suivent sont obtenus pour un maillage $N r \times N z$ de $150^{2}$. Le pas de temps considéré dans la plupart de solutions est de $\delta \tau=10^{-5}$. Il a été montré numériquement que toutes les solutions sont indépendantes du pas de temps pour des valeurs de $\delta \tau$ inférieures ou égales à cette valeur.

Le code de calcul a été validé avec les résultats bibliographiques concernant la convection naturelle. En régime permanent, les résultats sont comparés avec les résultats de De Vahl Davis [8]. En régime transitoire, nous avons validé notre programme avec la solution analytique obtenue par Carslaw et Jaeger [9] en régime diffusif, Leal et al. [10] et Sai et al. [11]. La comparaison de nos résultats avec ceux de la bibliographie montre un bon accord avec un maximum d'erreur inférieur à $3 \%$.

\section{Résultats et discussions}

Dans un premier temps nous analyserons l'effet de la courbure sur le transfert de chaleur en régime permanent et transitoire. Un chauffage est appliqué initialement au domaine. Dans toute notre étude, on considère la cavité de rapport de forme égal à $1(A=1)$ remplie d'air $(\mathrm{Pr}=$ 0,71).

L'évolution temporelle du transfert de chaleur pour différentes courbures est représentée sur la figure 2 pour différents nombres de Grashof $\operatorname{Gr} \in\left\{10^{4}, 10^{5}, 10^{6}\right\}$. Comme la courbure induit différents transferts de chaleur dans l'état d'équilibre, le nombre de Nusselt $\mathrm{Nu} / \mathrm{Nu}_{\text {permanent }}$ présenté sur la figure 2 est normalisé par le transfert de chaleur en régime permanent. Le temps nécessaire pour atteindre le régime permanent est indépendant de la courbure et diminue avec le nombre de Grashof. Les transferts en régime permanent correspondant aux différentes courbures sont représentés sur la figure 3 où il est également reporté en trait 


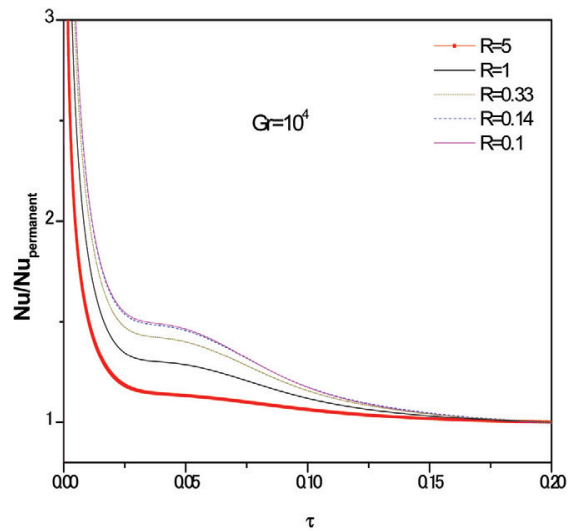

a)

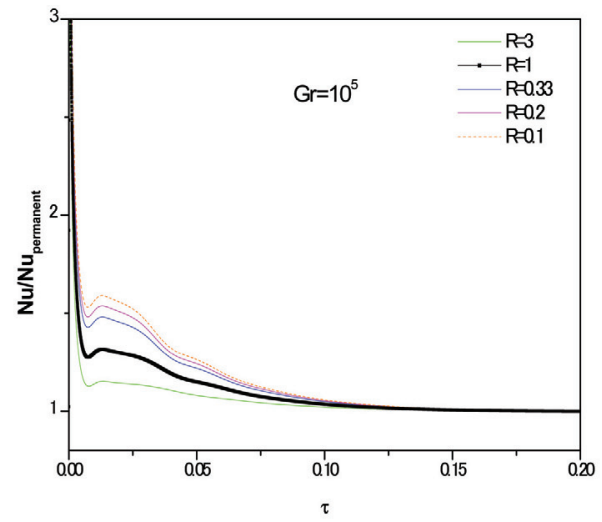

b)

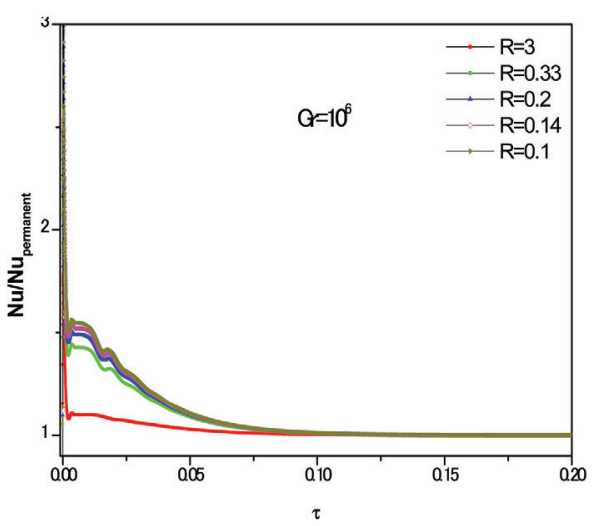

c)

Fig. 2. Évolution temporelle du nombre de Nusselt normalisé pour différentes courbures et différents nombres de Grashof $\mathrm{Gr}=10^{4}(\mathrm{a}), \mathrm{Gr}=10^{5}(\mathrm{~b})$ et $\mathrm{Gr}=10^{6}(\mathrm{c})$.

continu le transfert diffusif. Ce dernier est exprimé par $\left(\mathrm{Nu}_{\mathrm{i}}\right)_{\text {diffusive }}=\frac{R}{\ln (R+1)}$. On note l'accroissement du transfert convectif et diffusif avec la courbure ainsi que la constance du nombre de Nusselt pour les faibles courbures ce qui correspond au cas cartésien $(R=0)$. Ceci est la conséquence de la non-symétrie comme le montre le champ de la température sur la figure 4.

Avec l'accroissement de la courbure, la contribution diffusive croît, par contre la contribution convective atteint une valeur asymptotique quand la température au centre est voisine de la température froide. Cette asymptote concorde au cas de la convection naturelle sur les parois d'un cylindre en domaine infini. Ces précédentes tendances engendrent un transfert convectif d'autant plus proche du transfert conductif que la courbure est grande.

Comme l'une des conditions pour l'accroissement des transferts réside dans l'augmentation de la surface d'échange, on utilisera un bloc poreux. L'effet de la perméabilité du bloc poreux permettra de caractériser l'évolution temporelle des cas intermédiaires entre les cas extrêmes représentés par le cas d'un bloc solide et le cas $\mathrm{du}$ fluide.

On considère toujours la cavité remplie d'air $(\mathrm{Pr}=$ $0,71)$ de rapport de forme égal à l'unité $(A=1)$ pour

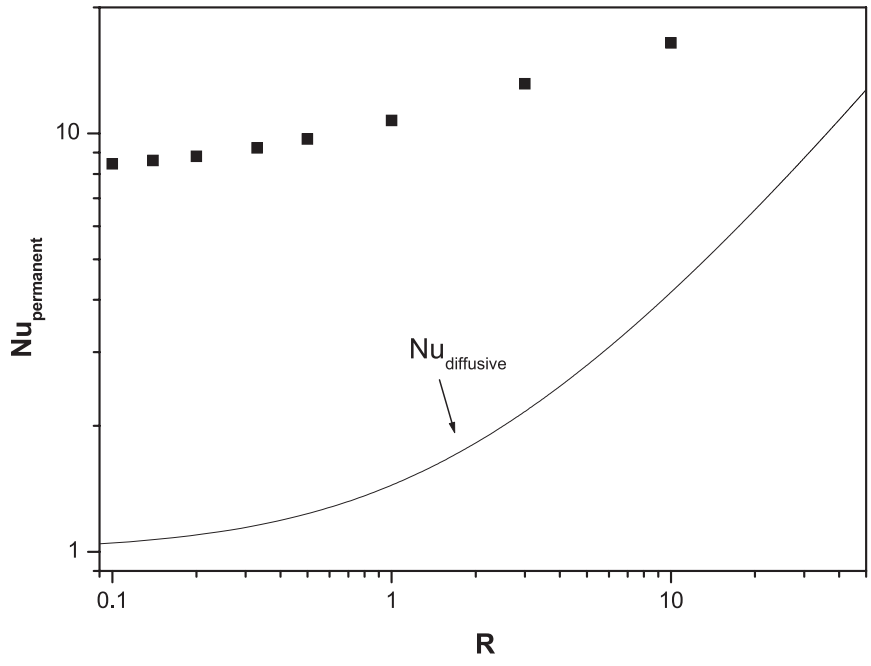

Fig. 3. Variation analytique (en trait continu) et numérique pour différentes courbures (pour $\mathrm{Gr}=10^{7}$ ).

$\mathrm{Gr}=10^{7}$ et on introduit l'obstacle à mi-hauteur de la paroi, de taille $e=h=0,1$.

La figure 5 représente l'évolution temporelle du nombre de Nusselt normé par sa valeur en régime 


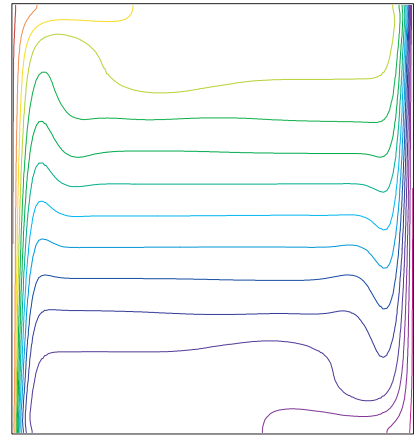

a)

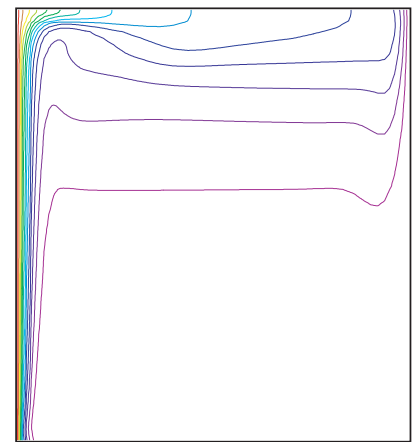

b)
Fig. 4. Champ de température (pour $\mathrm{Gr}=10^{7}$ ) pour une faible courbure (cas cartésien, $R \rightarrow 0$ ) a) et pour une grande courbure $(R=9)$ b).

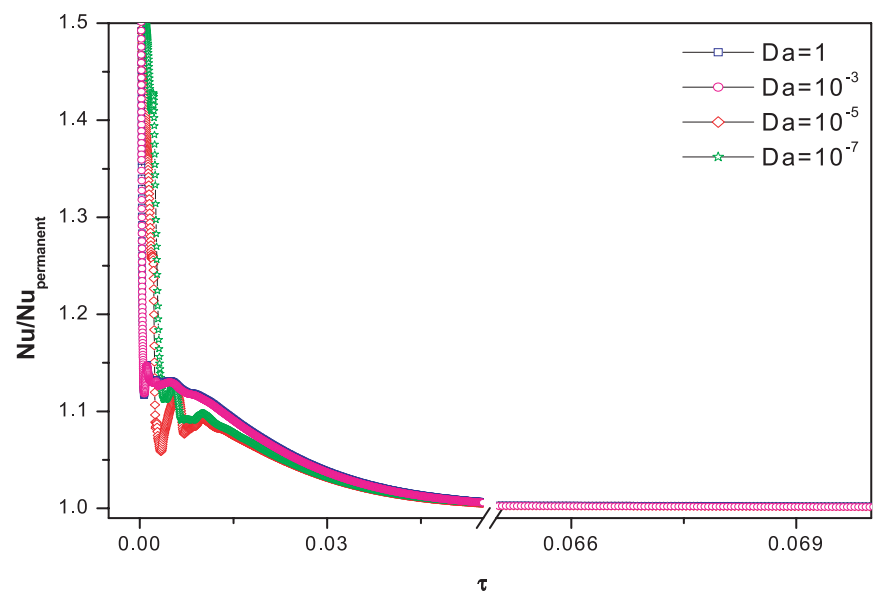

Fig. 5. Variation temporelle du nombre de Nusselt normé pour différents nombres de Darcy (pour $R=2$ et $\mathrm{Gr}=10^{7}$ ).

permanent pour différentes valeurs du nombre de Darcy du bloc isolant.

Aux premiers instants, pour des faibles valeurs de la perméabilité, nous remarquons une augmentation des transferts et des oscillations qui s'atténuent au fil du temps. Le temps nécessaire pour atteindre le régime permanent est de l'ordre de $\tau \approx 5 \times 10^{-2}$ pour ces faibles perméabilités.

Pour les grandes valeurs de la perméabilité, le régime permanent est atteint à partir de l'instant $\tau \approx 4,49 \times 10^{-2}$. L'utilisation des obstacles isolants solides ou perforés augmente le transfert aux premiers instants et retarde de $5 \%$ environ l'établissement du régime permanent.

La figure 6 représente la variation du nombre de Nusselt obtenue en régime permanent pour les différentes valeurs du nombre de Darcy. Nous pouvons caractériser les différentes situations : pour les très faibles valeurs du nombre de Darcy, la présence du bloc solide atténue les transferts de chaleur. Dans la zone allant de $\mathrm{Da}=10^{-5}$ à $10^{-3}$, le modèle de Darcy-Brinkman permet de quantifier l'accroissement résultant du possible écoulement à travers le bloc perméable. Pour Da $>10^{-3}$, on retrouve le cas asymptotique correspondant à la situation d'une cavité totalement fluide.

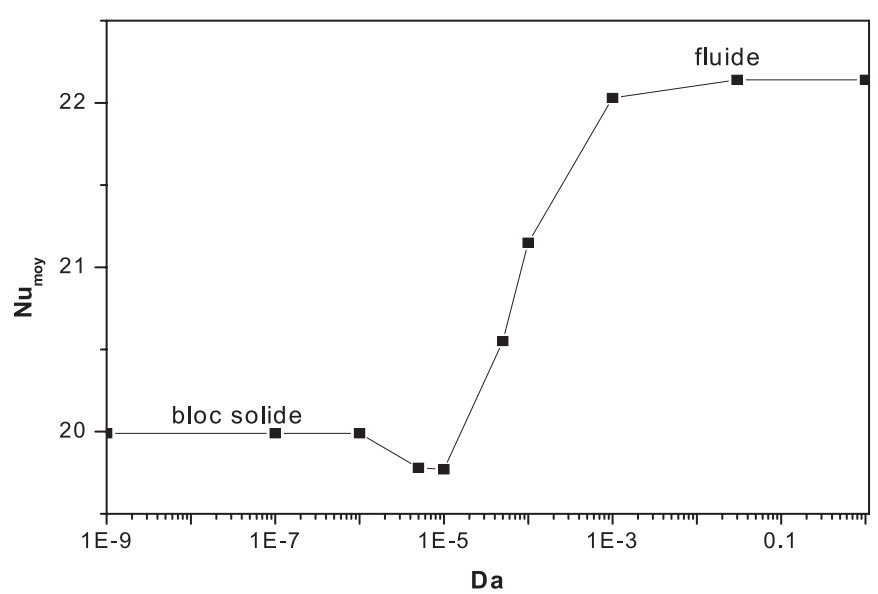

Fig. 6. Variation du nombre de Nusselt moyen en fonction du nombre de Darcy en régime permanent (pour $R=2$ et $\left.\operatorname{Gr}=10^{7}\right)$.

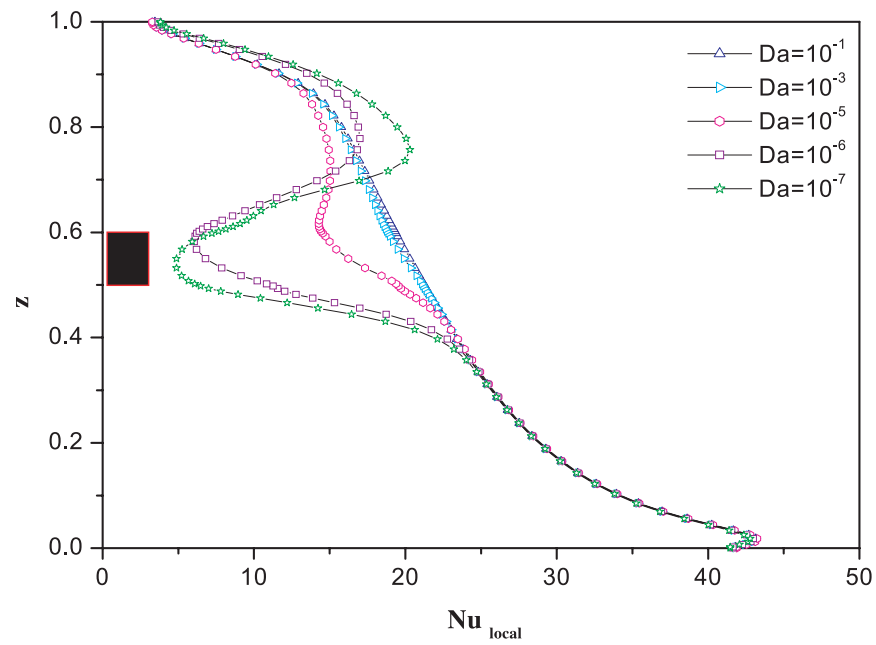

Fig. 7. Variation du nombre de Nusselt local le long de la paroi chaude pour différentes valeurs du nombre de Darcy (pour $R=2$ et $\mathrm{Gr}=10^{7}$ ).

Pour expliquer ce minimum du transfert de chaleur dans le second cas autour de $\mathrm{Da}=10^{-5}$, nous avons tracé le transfert local le long de la paroi chaude (Fig. 7). Le transfert diminue avec Darcy au niveau de l'obstacle puis s'accroît après le bloc. Cet accroissement qui amoindrit la diminution globale du nombre de Nusselt n'est pas observé pour $\mathrm{Da}=10^{-5}$.

Cette stagnation du transfert à $\mathrm{Da}=10^{-5}$ est la conséquence directe du faible écoulement qui passe à travers le bloc où le fluide se réchauffe (Fig. 8). Cette figure représente la variation de la vitesse à mi-hauteur de la paroi pour différents nombres de Darcy, on y retrouve la dissymétrie de l'écoulement pour les faibles valeurs du nombre de Darcy. Pour ces faibles nombres de Darcy, l'écoulement à travers le bloc s'amoindrit et une partie de l'écoulement contourne le bloc. Sur le cylindre extérieur, l'écoulement n'est pas affecté pour les différents nombres de Darcy considérés. 


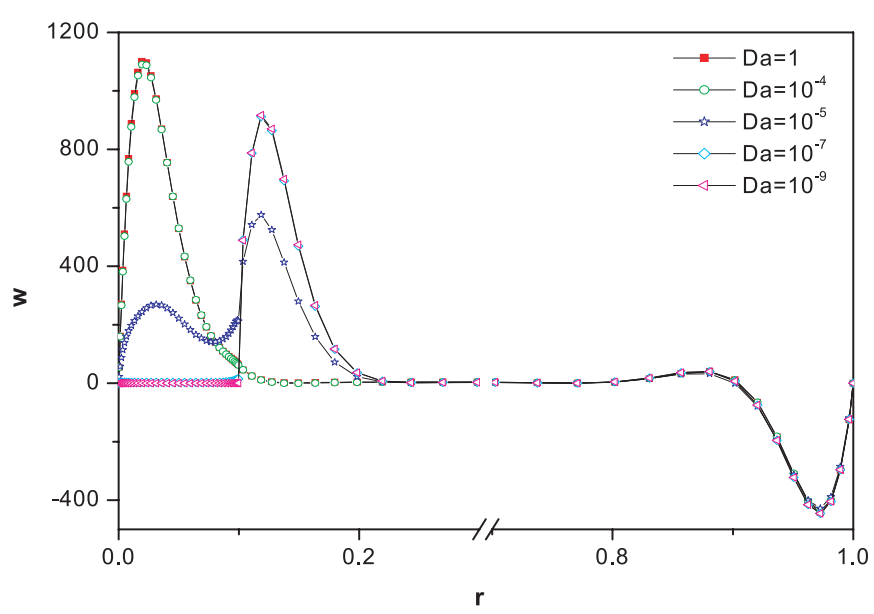

Fig. 8. Profils des vitesses pour différents nombres de Darcy (pour $R=2$ et $\mathrm{Gr}=10^{7}$ ).
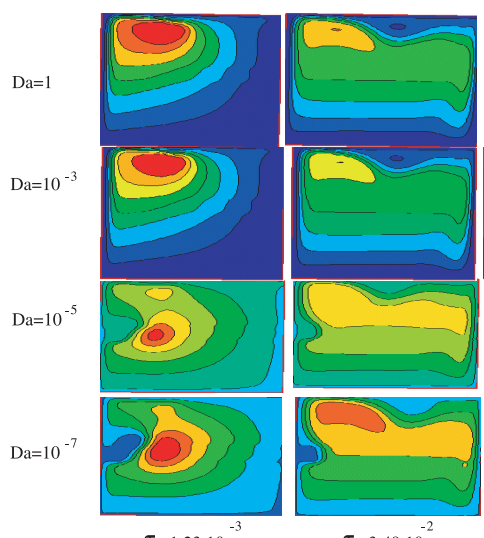

$\tau=3,49.10^{-2}$
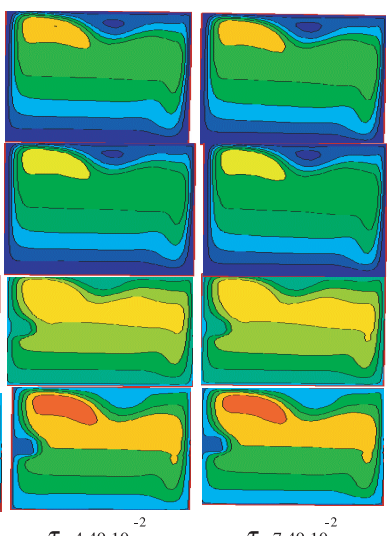

Fig. 9. Champs dynamiques à différents instants et à différents nombres de Darcy (pour $R=2$ et $\mathrm{Gr}=10^{7}$ ).

Ces observations sont également illustrées sur la figure 9 représentant les champs dynamiques à différents instants et pour différentes valeurs du nombre de Darcy.

\section{Conclusion}

Dans la présente étude, nous avons étudié l'effet temporel sur le transfert thermique pour différentes configurations. Nous avons montré que le temps nécessaire pour atteindre le régime permanent est indépendant de la courbure et diminue avec l'augmentation du nombre de Grashof. Ce transfert augmente dans le cas de la cavité annulaire. Pour cette dernière nous avons introduit un bloc poreux. Nous avons montré que l'utilisation des obstacles isolants solides ou perforés augmente le transfert aux premiers instants et retarde de $5 \%$ environ l'établissement du régime permanent. Nous avons montré aussi que l'utilisation des obstacles perforés permet l'augmentation du transfert par rapport à des obstacles pleins. Cependant, la conductivité dans l'obstacle poreux considérée dans cette étude est faible et égale à celle du fluide. L'autre résultat en cours de finalisation réside dans la modification de la conductivité de l'obstacle permettant de quantifier l'effet des obstacles poreux conducteurs.

\section{Références}

[1] G. De Vahl Davis, R.W. Thomas, Natural convection between concentric vertical cylinders, High speed computing in Fluid Dynamics Physics of Fluids, Supplement II, (1969) 198-207

[2] V. Prasad, F.A. Kulacki, Free convection heat transfer in a liquid-filled vertical Annulus, J. Heat Transfer 107 (1985) 596-602

[3] R. Kumar, M.A. Kalam, Laminar thermal convection between vertical coaxial isothermal cylinders, Int. J. Heat and Mass Transfer 34 (1991) 513-524

[4] V. Prasad, Numerical study of natural convection in a vertical porous annulus with constant heat flux on the inner wall, Int. J. Heat and Mass Transfer 29 (1986) 841-853

[5] J. Patterson, J. Imberger, Unsteady natural convection in a rectangular cavity, J. Fluid. Mech. 1000 (1980) $65-86$

[6] J.M. Hyun, J.W. Lee, Numerical solutions for transient natural convection in a square cavity with different sidewall temperature, Int. J. Heat and Fluid Flow 10 (1989) 146-151

[7] S. Patankar, Numerical Heat Transfer and Fluid Flow, Hemisphere, New York, 1980

[8] G. De Vahl Davis, Natural convection of air in a square cavity: a bench mark numerical solution, Int. J. Num. Meth. in Fluids 3 (1983) 249-264

[9] H.S. Carslaw, J.C. Jaeger, Conduction of heat in solids, Oxford Science Publications, Second edition, 1959

[10] M.A. Leal, H.A. Machado, R.M. Cotta, Integral transform solutions of transient natural convection in enclosures with variable fluid properties, Int. J. Heat and Mass Transfer 43 (2000) 3977-3990

[11] B.V.K.S. Sai, K.N. Seetharamu, P.A.A. Narayana, Solution of transient laminar natural convection in a square cavity by an explicit finite element scheme, Num. Heat Transfer Part A 25 (1994) 412-422 